\title{
COMPORTAMENTOS DE RISCO PARA AS DOENÇAS SEXUALMENTE TRANSMISSÍVEIS EM ADOLESCENTES ESCOLARES DE BAIXA RENDA
}

\author{
RISK BEHAVIORS FOR SEXUAL TRANSMITTED DISEASE IN LOW INCOME SCHOLAR \\ ADOLESCENTS

\section{COMPORTAMIENTOS DEL RIESGO PARA LAS ENFERMEDADES SEXUAL TRANSMISSIBLE EN ADOLESCENTES ESTUDIANTES DE BAJA RENTA}

\section{Pricilla Diniz Bonfim Silva ${ }^{1}$, Michele Dias da Silva Oliveira ${ }^{2}$, Marcos André de Matos ${ }^{1}$, Viviane Rodrigues Tavares ${ }^{1}$, Marcelo Medeiros ${ }^{3}$, Sandra Brunini ${ }^{4}$, Sheila Araújo Teles ${ }^{5}$}

\begin{abstract}
RESUMO: Os adolescentes costumam serem vulneráveis a comportamentos de risco para aquisição de doenças sexualmente transmissíveis (DST). O início precoce da atividade sexual, a multiplicidade de parceiros, o uso esporádico de preservativo, o consumo de bebida alcoólica e drogas ilícitas têm sido considerados preditores para as DST. Para identificar os comportamentos de risco para as DST, 223 adolescentes escolares de uma região de baixa renda adjacente à área metropolitana de Goiânia foram entrevistados durante novembro e dezembro de 2003. Verificou-se que neste grupo a média de idade da primeira relação sexual foi de 14,9 anos, sendo a média de parceiro sexual igual a quatro. $O$ uso irregular ou não uso de preservativos foi relatado por $44,1 \%$ dos indivíduos. Quase a totalidade $(80,7 \%)$ dos adolescentes relatou consumo de bebida alcoólica e $13,9 \%$ uso de drogas ilícitas. Verificou-se ainda uma diferença estatisticamente significativa destes comportamentos em relação ao gênero. Gravidez foi reportada por $14 \%$ das adolescentes. Os achados deste estudo sugerem um elevado risco de doenças sexualmente transmissíveis e evidenciam a necessidade urgente de programas de saúde para este grupo alvo.
\end{abstract}

PALAVRAS-CHAVE: Saúde do Adolescente; Saúde Escolar; Doenças Sexualmente Transmissíveis.

ABSTRACT: Adolescents have been vulnerable to sexually transmissible diseases (STD) risk behaviors. The beginning of the sexual activity, the multiplicity of partners, the irregular use of condom, the consumption of alcoholic beverage and illicit drugs have been considered predictors for the STD. in order to identify the risk behaviors for STD in youth of an low income region adjacent the metropolitan area of Goiânia city, 223 schoolar adolescents were interviewed during November and December 2003. The means age of the first sexual intercourse was of 14.9 years and the average of partners along the life was 4.0. Of the total individuals, $44.1 \%$ reported irregular use or not use of condoms during sexual intercourse. Almost the totality $(80.7 \%)$ mentioned alcohol consumption and $13.9 \%$ illicit drugs. There was a statistical difference among these risk behaviors and sex. Pregnant was reported by $14 \%$ of the female adolescents. The findings of this study suggest a high vulnerability for sexually transmissible diseases, and highlight the urgent necessity of health programs for this target group.

KEYWORDS: Teen Health; School Health; Sexually Transmitted Diseases.

RESUMEN: LoS adolescentes costrubran ser vulnerables a comportamientos de riesgo para aquisición de dolencias sexualmente transmisibles (DST). El inicio precoz de la actividad sexual, la multiplicidad de parceros, el uso esporadico de preventivo, el consumo de bebída alcohólica y drogas ilícitas son considerados preditores para las DST. Para reconecer los comportamientos de riesgo para las DST, 223 adolescentes escolares de una región del envuelta de Goiânia fueron entrevistados durante noviembro y diciembre de 2003. La media de edad de la primera relación sexual fue de 14,9 años, sendo que la media de parceros fue de 4,0. Del total de los entrevistados, $44,1 \%$ citaron uso irregular o no uso de preventivos. Casi la totalidad (80.7\%) mencionó la consumición del alcohol y $13.9 \%$ de las drogas ilícitas. Había una diferencia estadística entre estos comportamientos y sexo. Embarazo fue divulgado por $14 \%$ de las adolescentes. Los resultados de este estudio sugieren una alta vulnerabilidad para las enfermedades sexual transmissible, y destacan la necesidad urgente de los programas de la salud para este grupo.

1 Acadêmico(a) da Faculdade de Enfermagem (FEN) da Universidade Federal de Goiás (UFG). Goiânia, (GO).

2 Enfermeira. Mestranda do Programa de Pós Graduação - Mestrado em Enfermagem - da Faculdade de Enfermagem da Universidade federal de Goiás. Professora Substituta da FEN/UFG.

3 Enfermeiro. Doutor em Enfermagem. Professor Adjunto da Faculdade de Enfermagem da Universidade Federal de Goiás.

4 Enfermeira. Mestre em Patologia Tropical. Doutoranda no Programa de Pós Graduação - Doutorado em Enfermagem - da Escola de Enfermagem de Ribeirão Preto da Universidade de São Paulo. Professora Assistente da Faculdade de Enfermagem da Universidade Federal de Goiás

5 Enfermeira. Doutora em Patologia Tropical. Professor Adjunto da Faculdade de Enfermagem da Universidade Federal de Goiás. Rua 227, quadra 68, s/n, FEN/UFG, CEP: 74605-080; Goiânia-Goiás, e-mail: sheila@fen.ufg.br 
PALABRAS CLAVE: Salud de los adolescentes; Salud Escolar; Enfermedades Sexualmente

\section{INTRODUÇÃO}

Estima-se que, a cada ano, quatro milhões de jovens tornam-se sexualmente ativos no Brasil (SANTOS \& SANTOS, 2004). De acordo com a Organização Mundial de Saúde (OMS), ocorrem no País cerca de 12 milhões de doenças sexualmente transmissíveis (DST) ao ano. Ainda, um terço destas em indivíduos com menos de 25 anos (BRASIL, 2004).

As DST têm sido causa de salpingites, uretrites e vulvovaginites, podendo causar ainda infertilidade, gravidez ectópica e câncer uterino. Mais, estas infecções potencializam o risco de infecção pelo HIV (DONOVAN 2004).

Os adolescentes são vulneráveis a comportamentos de risco para aquisição de doenças sexualmente transmissíveis como multiplicidade de parceiros sexuais, uso irregular de preservativo, consumo de bebida alcoólica e drogas ilícitas. Ademais, os mesmos sentem-se invulneráveis às doenças, se expondo a risco sem prever conseqüências (MURPHY et al. 2001).

Para ELLEN et al (1996), os esforços para reduzir as taxas de doenças sexualmente transmissíveis em adolescentes devem incluir intervenções nas escolas, comunidade e serviços de saúde que despertem nos jovens a percepção de risco pessoal para aquisição destas infecções.

Um estudo descritivo é o primeiro passo para se estabelecer a situação de base para posteriores avaliações da efetividade de medidas de intervenção. Nesta investigação, identificaram-se fatores que favorecem a transmissão de DST em adolescentes escolares de uma região de baixa renda adjacente à área metropolitana de Goiânia. Os resultados deste estudo contribuirão para a construção de estratégias eficazes de prevenção destas doenças, que são importantes causas de morbidade em adolescentes.

\section{MATERIAL E MÉTODOS}

Estudo transversal, realizado em novembro e dezembro de 2003, em 223 adolescentes de 15 a 19 anos, sexualmente ativos, matriculados em duas
Transmisibles.

escolas públicas de uma região de baixa renda, limítrofe entre as cidades de Aparecida de Goiânia e Goiânia, que possuíam a segunda fase do ensino fundamental e médio.

Após esclarecimentos sobre o projeto, autorização dos responsáveis pelos adolescentes ou dos próprios adolescentes (idade igual ou superior a 18 anos) procedeu-se à entrevista, face a face, utilizando-se um roteiro estruturado contendo dados pessoais (gênero, idade, renda familiar e naturalidade) e fatores associados as DST (uso de drogas ilícitas e bebida alcoólica, gravidez, idade da $1^{\text {a }}$ relação sexual, número de parceiros e uso de preservativo). Todas as entrevistas foram realizadas em local privado, sendo assegurado aos adolescentes o caráter confidencial dos dados.

Os dados das entrevistas foram digitados em microcomputador e analisados no programa "Epilnfo 6" versão 6.04, desenvolvido pelo "Centers for disease Control and Prevention", Atlanta, GA, Estados Unidos da América. Realizou-se a análise descritiva por meio de distribuição de freqüências, cálculo das médias e desvio padrão. As variáveis categóricas foram comparadas utilizando-se o teste de qui-quadrado, e as variáveis contínuas o teste $\mathrm{H}$ de Kruskal-Wallis. Quando apropriado o teste de Fisher foi utilizado. Em todas as análises, estabeleceu-se um intervalo de $95 \%$ de confiança.

Este projeto foi aprovado pelo Comitê de Ética em Pesquisa do Hospital da Santa Casa de Misericórdia de Goiânia.

\section{RESULTADOS}

A Tabela 1 apresenta as características sóciodemográficas dos adolescentes escolares investigados. A média de idade foi de 17,1 anos com desvio padrão de 1,3 ano. Quanto ao gênero, 48,9\% eram do gênero feminino e $51,1 \%$ do gênero masculino. Com relação à naturalidade, 51,6\% dos indivíduos eram naturais do estado de Goiás e 48,4\% de outros estados. Somente $9,4 \%$ dos adolescentes relataram renda superior a três salários mínimos (s.m.).

Tabela 1 - Características sócio-demográficas dos escolares adolescentes de uma região de baixa renda, 2003.

Características

Média de idade 17,1 anos $( \pm 1,3)$
(\%)

\section{Gênero}

Feminino
$\mathbf{N}$ 
Masculino

114

115

108

160

18

Sem informação 24

A média de idade da primeira relação foi de 14,9 anos $( \pm 1,6)$ (Figura 1). Contudo, uma diferença significativa foi observada entre os adolescentes do

Figura 1 - Média de idade da primeira relação sexual em escolares adolescentes de uma região de baixa renda, da cidade de Goiânia, 2003. gênero feminino e masculino $(14,5 \pm 1,6$ vs. $15,4 \pm 1,5$; $\mathrm{p}<0,05)$.

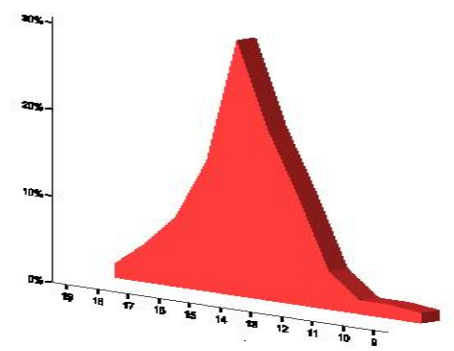

Em relação ao número de parceiros, os jovens relataram uma média de 4,0 parceiros sexuais. Entretanto, os rapazes reportaram uma média de 6,0 parceiras $( \pm 5,7)$, enquanto as moças 1,9 parceiro ( \pm $1,5)$, sendo esta diferença estatisticamente significativa $(p=0,05)$ (Tabela 2).

Em relação ao uso de preservativos, consumo de bebida alcoólica e drogas ilícitas, houve também diferenças significativas em relação ao gênero dos adolescentes. Do total de indivíduos do gênero masculino, $58,8 \%$ afirmaram que usavam

Tabela 2 - Distribuição dos adolescentes escolares de uma região de baixa renda, segundo comportamento de risco e sexo, 2003

\begin{tabular}{|c|c|c|c|c|c|}
\hline \multirow{3}{*}{ Comportamento } & \multicolumn{4}{|c|}{ Gênero } & \multirow{3}{*}{ Valor de $p$} \\
\hline & \multicolumn{2}{|c|}{ Masculino } & \multicolumn{2}{|c|}{ Feminino } & \\
\hline & $\mathrm{n}=119$ & $\%$ & $\mathrm{n}=114$ & $\%$ & \\
\hline Média de idade da $1^{a}$ relação sexual & \multirow{2}{*}{\multicolumn{2}{|c|}{$\begin{array}{c}14,5( \pm 1,6) \\
6,0( \pm 5,7)\end{array}$}} & \multirow{2}{*}{\multicolumn{2}{|c|}{$\begin{array}{c}15,4( \pm 1,5) \\
1,9( \pm 1,5)\end{array}$}} & $<0,05$ \\
\hline Média de parceiros sexuais & & & & & $<0,05$ \\
\hline \multicolumn{6}{|l|}{ Uso regular de preservativos } \\
\hline Sim & 73 & $(61,3)$ & 51 & $(44,7)$ & \\
\hline Não & 41 & $(34,4)$ & 57 & $(50,0)$ & $<0,05$ \\
\hline \multicolumn{6}{|l|}{ Consumo de bebida alcoólica } \\
\hline Não & 15 & $(12,6)$ & 15 & $(13,1)$ & \\
\hline Sim & 99 & $(83,1)$ & 81 & $(71,0)$ & $<0,05$ \\
\hline
\end{tabular}

preservativos regularmente durante intercurso sexual, enquanto somente $41,8 \%$ das adolescentes reportaram esta prática $(p<0,05)$. O consumo de bebida alcoólica foi citado por $86,8 \%$ rapazes enquanto $74,3 \%$ das adolescentes relataram este comportamento $(p<0,05)$. O uso de drogas ilícitas foi relatado por $19,3 \%$ dos escolares do gênero masculino ao passo que somente $8,2 \%$ das estudantes referiram uso de drogas $(p<0,05)$ (Tabela 2$)$. Já gravidez foi referida por $14,0 \%$ das jovens entrevistadas. 
Uso de drogas ilícitas

Não

Sim
$(18,5)$

$(77,3)$

$\begin{array}{cl}100 & (87,7) \\ 9 & (7,9)\end{array}$

$<0,05$

\section{DISCUSSÃO}

Estudos sugerem que a idade da primeira relação, a multiplicidade de parceiros (homo ou heterossexual) e a desconsideração ao uso de preservativos nas relações sexuais são importantes comportamentos de risco para doenças sexualmente transmissíveis (CARRET et al. 2004; MEHEUS, 2000; TAQUETTE et al. 2005). Ainda, para alguns a baixa condição socioeconômica é um fator preditor para o início precoce da vida sexual (FELICE et al, 1999). De fato, neste estudo, verificou-se o início precoce da vida sexual em adolescentes de baixa renda, ratificando um estudo prévio, de base populacional, realizado na periferia de Goiânia onde mais da metade das adolescentes investigadas referiram a primeira relação sexual aos 15 anos de idade ou menos (VIEIRA et al 2004). Ainda, como observados em outros estudos brasileiros (TAQUETTE et al, 2004; TRAJMAM et al, 2003), os rapazes iniciaram a vida sexual mais precocemente que as adolescentes e referiram mais promiscuidade sexual.

Em relação à média de parceiros sexuais, verificou-se uma diferença estatisticamente significativa entre as médias de parceiros em adolescentes do gênero masculino e feminino ratificando, assim, os resultados de TRAJMAN et al (2003) e TAQUETTE et al (2004) em adolescentes do Rio de Janeiro.

Do total de adolescentes, $55,6 \%$ relataram uso regular de preservativos. Entretanto, houve uma diferença em relação ao gênero. Enquanto somente $47,2 \%$ das mulheres assumiram o uso regular de preservativos, $64 \%$ dos homens referiram esta prática em todas as relações sexuais $(p<0,05)$. Outros estudos também têm mostrado um índice menor de uso consistente do preservativo em mulheres quando comparados com os homens (TAQUETTE et al, 2004). Para alguns autores, o baixo uso de preservativos deve-se as dificuldades de negociação do uso de preservativos entre parceiros durante as relações sexuais, principalmente em camadas mais pobres da sociedade (TAQUETTE et al 2003). Em Goiânia, um estudo prévio mostrou que somente $20,5 \%$ das adolescentes de uma região de baixa renda referiram uso regular de preservativos (VIEIRA et al, 2004).

Investigações têm mostrado uma associação positiva entre uso de drogas e/ou álcool e DST (SANTELLI et al, 2004; TAQUETTE et al, 2005). O uso de drogas ilícitas foi relatado por $13,9 \%$ dos investigados e o consumo de bebida alcoólica por quase a totalidade (80,7\%). Estes foram significativamente mais freqüentes em homens do que mulheres $(p<0,05)$. TAQUETTE et al (2004) também encontraram uma associação positiva entre uso de drogas ilícitas e gênero masculino. O uso destas substâncias favorece a adoção de práticas irresponsáveis como o compartilhamento de seringas, agulhas e outros materiais durante o consumo, bem como relações sexuais desprotegidas. Tais comportamentos os tornam vulneráveis as infecções por doenças sexualmente transmissíveis (MEHEUS, 2000).

Nesta investigação, $14,0 \%$ das adolescentes escolares relataram gravidez. Este índice foi inferior $(42,1 \%)$ ao encontrado por VIEIRA et al (2004). Contudo, deve-se salientar que $53,3 \%$ das adolescentes investigadas por estes autores tinham menos de oito anos de escolaridade, um fator que parece contribuir para a gravidez precoce (FELICE et al, 1999).

Os achados deste estudo demonstram o despreparo dos adolescentes para uma vida sexual sadia e, o risco destes para doenças sexualmente transmissíveis, o que reforça a importância de programas educativos para a população de adolescentes, principalmente para aqueles de baixa renda, que residem em áreas de maior risco social e que parecem estar mais susceptíveis a comportamento que favorecem a disseminação de DST.

\section{REFERÊNCIAS BIBLIOGRÁFICAS}

BRASIL. Ministério da Saúde. Dados e pesquisas em DST e Aids. Disponível em: http://www.aids.gov.br/final/dados/DST.htm . [acesso em 18 nov. de 2004].

CARRET, M. L. et al. Sexually transmitted diseases symptoms in adults: prevalence and risk factors Revista de Saúde Pública. v. 38, p. 7-84, 2004.

DONOVAN, B. Sexually transmissible infections other than HIV. The Lancet. v. 363, p. 545-556, 2004

ELLEN, M. J. et al. Adolescents Perceived Risk for STDs and HIV Infection. Journal of Adolescents Health, v. 18, p.177-181, 1996.

FELICE, M. E. et al. Adolescent pregnancy--current trends and issues: 1998 American Academy of Pediatrics Committee on Adolescence, 1998-1999. Pediatrics. v.103, p. 516-520, 1999.

MEHEUS, A. Teenagers lifestyle and the risk of exposure to hepatitis B virus. Vaccine, v.18, Suppl 1, p. 26-29. 2000.

MURPHY, D. A. et al. No change in health risk behaviors over time among HIV infectd adolescents in 
care: role of psychological distress. Journal of adolescent Health. v. 29S, p. 57-63, 2001.

SANTELLI, J. S. et al. Initiation of sexual intercourse among middle school adolescents:The influence of psychosocial factors. Journal of adolescent and Health. v. 34, p. 200-208, 2004.

SANTOS, L. V.; SANTOS, E. C. Adolescentes, jovens e aids no Brasil. Disponível em: http://www.aids.gov.br//prevencao/adolescentes.htm. [acesso em 18 nov. de 2004].

TAQUETTE, S. R. et al. A relação entre as características sociais e comportamentais da adolescente e as doenças sexualmente transmissíveis. Revista da Associação Médica Brasileira. v. 51, p. 148152, 2005.

TAQUETTE, S. R. et al. Violent relationship in young people and STD/AIDS risk. Cadernos de Saúde Pública. v. 19, p. 1437-1444, 2003.
TAQUETTE, S.R. et al. Sexually transmitted diseases and gender: a cross-sectional study with adolescents in Rio de Janeiro Cadernos de Saúde Pública. v. 20, p.282-290, 2004.

TRAJMAN, A. et al. Knowledge about STD/AIDS and sexual behavior among high school students in Rio de Janeiro, Brazil. Cadernos de Saúde Pública, v. 19, p. 127-133, 2003.

VIEIRA. M.A.S. et al. Fatores associados ao uso de preservtivo em adolescentes do gênero feminino no município de Goiânia. Jornal Brasileiro de Doenças Sexualmente Transmissíveis. v. 16, p. 77-83, 2004.

Texto original recebido em 19/08/2005

Publicação aprovada em 30/08/2005 